\title{
Expression of IL-2R, IL-4R, IL-6R on peripheral blood lymphocytes in systemic lupus erythematosus and correlation with disease activity: a prospective study
}

\begin{abstract}
Aims-To study the expression of interleukin-2 receptor (IL-2R), interleukin-4 receptor (IL-4R) and interleukin-6 receptor (IL-6R) on peripheral blood lymphocytes (PBL) in patients with systemic lupus erythematosus (SLE); to correlate the level of expression of these receptors with SLE disease activity.

Methods-Peripheral blood lymphocytes were studied by a high sensitivity flow cytometry technique using monoclonal antibodies directed against CD25 (IL-2R $\alpha$ chain), CD122 (IL-2R $\beta$ chain), CD124 (IL-4R), and CD126 (IL-6R). SLE disease activity was scored using the SLE Disease Activity Index, C3 and C4 concentrations, anti-dsDNA level, and absolute lymphocyte count.
\end{abstract}

Results-Compared with normal controls, PBL from patients with SLE had a higher percentage of CD25+ cells (median $20.8 \% v 16.5 \%$ ) and a lower percentage of CD122+ cells (median $13.1 \%$ v $22.4 \%$ ). The difference in $\mathrm{CD} 122+$ cells was greater in the $\mathrm{CD} 122^{\text {weak }}$ population than the $\mathrm{CD}_{122^{\text {strong }}}$ (natural killer cell) population. The percentages of CD124+ and CD126+ PBLs in patients with SLE and controls were similar. On CD25+ cells, the relative antigenic level of the IL-2R $\alpha$ chain was significantly higher in patients with SLE (median $2.01 v$ 1.81). The relative antigenic levels of $\mathrm{CD} 122+$, CD124+ and CD126+ cells were similar in patients and controls. Neither the percentages nor the relative antigenic levels of all of these cytokine receptors were correlated with any of the parameters of disease activity.

Conclusion-Lymphocyte activation in patients with SLE was evident from the increase in CD25 expression on PBL, with a reciprocal decrease in CD122 expression. As the expression of IL-2R, IL-4R, IL-6R did not correlate with disease activity, it seems that these cytokine/receptor systems do not play a direct role in disease activation in SLE.

(f Clin Pathol 1996;49:660-663)

Keywords: cytokine receptors, systemic lupus erythematosus, peripheral blood lymphocytes.
Systemic lupus erythematosus (SLE) is characterised by several functional abnormalities of the immune system, including impaired $\mathrm{T}$ cell responses and B cell hyper-reactivity. Abnormalities of the interleukin-2/interleukin-2 receptor (IL-2/IL-2R) system have been described previously in these patients-for example, increased serum concentrations of $\mathrm{O}$ soluble IL-2R,,$^{1-5}$ increased cellular expression of the Tac (CD25) antigen, ${ }^{6-8}$ and impaired $D$ production of IL-2 in vitro. ${ }^{2-7}$ Interleukin-4 (IL-4) and interleukin-6 (IL-6) were initially $\stackrel{\text { ( }}{\rightarrow}$ identified as B cell growth and differentiation $\vec{\theta}$ factors. It is now known that the main of physiological function of IL-4 is as a regulator of allergic diseases; IL- 6 causes hepatocytes to synthesise several acute phase proteins. The role of IL- 4 and IL- 6 and their corresponding receptors in autoimmune diseases is not known but there is evidence suggesting these $\mathrm{T}_{\mathrm{H}} 2 \stackrel{\Rightarrow}{\Rightarrow}$ cytokines are involved in promoting and perpetuating B cell hyperactivity and autoantibody formation. ${ }^{9}$ In the present study, we sought to examine the expression of IL-2R, IL-4R, IL-6R on peripheral blood lymphocytes (PBL) of patients with SLE in comparison with normal individuals, using a sensitive immunofluorescence technique that detects low antigenic expression generally missed on conventional staining. The expression of these receptors on PBL was correlated with SLE disease activity. To measure disease activity, we have chosen the SLE Disease Activity Index $(\text { SLEDAI })^{10}$ which consists of 24 weighted clinical and laboratory parameters. Other $\mathrm{W}$ markers of SLE disease activity, including C3, श anti-dsDNA, and the absolute lymphocyte count were also measured for comparison.

\section{Methods}

Sixty nine (eight men) consecutive patients from the Department of Medicine, University of Hong Kong, who met four or more of the 8 criteria of the American College of Rheumatology for the classification of SLE, were studied. ${ }^{11}$ At the time of study, all patients were ? receiving no more than $15 \mathrm{mg}$ prednisolone; some were also taking $50-100 \mathrm{mg}$ azathioprine. There was no difference in drug treatment between the patients with active and inactive disease because the former were studied prior to the augmentation of their steroid 
Table 1 Median (first and third quartiles) of percentage of peripheral blood lymphocytes that express CD25, CD122, $C D 124$, and CD126

\begin{tabular}{lllll}
\hline & $C D 25$ & $C D 122$ & $C D 124$ & $C D 126$ \\
\hline Normal & $16.5(14.8-20.0)$ & $22.4(14.05-26.83)$ & $7.9(6.08-9.7)$ & $26.55(22.43-33.9)$ \\
SLE (overall) & $20.8(16.9-25.3)$ & $13.1(8.8-18.9)$ & $7.5(4.2-9)$ & $25.2(18-29.1)$ \\
SLE (SLEDAI < 5) & $20.3(15.2-23.5)$ & $11.25(8.35-17.3)$ & & \\
SLE (SLEDAI $>9)$ & $16.9(15.2-23.5)$ & $13.1(12.85-19.4)$ & & \\
\hline
\end{tabular}

Table 2 Median (first and third quartiles) of the relative antigenic level of CD25, CD122, CD124, and CD126 expressed on peripheral blood lymphocytes

\begin{tabular}{lllll}
\hline & CD25 & CD122 & CD124 & $C D 126$ \\
\hline Normal & $1.81(1.62-2.01)$ & $1.46(1.18-1.62)$ & $1.34(1.07-1.49)$ & $1.39(1.14-1.57)$ \\
SLE (overall) & $2.01(1.7-2.18)$ & $1.35(1.2-1.66)$ & $1.28(1.16-1.39)$ & $1.39(1.27-1.52)$ \\
SLE (SLEDAI < 5) & $2.04(1.79-2.18)$ & $1.23(1.16-1.41)$ & & \\
SLE (SLEDAI $>9)$ & $1.87(1.37-2.19)$ & $1.55(1.43-1.72)$ & & \\
\hline
\end{tabular}

dosage. For comparison, 51 healthy volunteers of similar age and sex distribution were included.

DISEASE ACTIVITY

The SLEDAI ${ }^{10}$ was used to assess the disease activity of each patient during the study. Clinical parameters were scored by two of the authors who followed the progress of all patients. Anti-dsDNA antibodies were assayed by ELISA using the International Reference Standard for anti-dsDNA antibody. C3 and C4 concentrations were measured using nephelometry.

Lymphocytes expressing the cytokine receptors were studied using a high sensitivity flow cytometry technique, as described previously. ${ }^{12}$ Briefly, peripheral blood mononuclear cells were obtained by Ficoll-Hypaque sedimentation. The cells were incubated with a mouse IgG monoclonal antibody, the binding of which was detected after washing with a biotinylated horse anti-mouse IgG (Vector Laboratories, Burlingame, California, USA) followed by Streptavidin-phycoerythrin (Dako, Glostrup, Denmark). The monoclonal antibodies used included CD25 (from H Zola), CD122 (Immunotech, Marseille, France), CD124 (Immunotech), and CD126 (Serotec, Oxford, UK) which bind the IL-2R $\alpha$ chain, the IL-2R $\beta$ chain, IL-4R, and IL-6R, respectively. The same batch of reagents was used throughout the study. For isotypic control, a mouse monoclonal antibody of the same isotype but not reacting with human lymphocytes, was used. After staining, the cells were analysed in a Coulter Profile II flow cytometer. Only cells with forward and side scatter characteristics of lymphocytes were gated. The cut-off level was set to include $2 \%$ of the isotypic control cells. The percentage of positive cells above this cutoff level and the mean fluorescence channel number were recorded.

QUALITY CONTROL

Optical alignment of the flow cytometer was set daily using DNA check standard beads (Coulter, Hialeah, Florida, USA). The machine settings were counter checked using CaliBRITE standard beads (Becton Dickinson, San Jose, California, USA). These beads gave a mean (SD) fluorescence level of 9.39 (0.10) between assays. Frozen mononuclear cells were stained in parallel with test samples during each assay.

STATISTICS

Two sample comparisons were performed by the Wilcoxon technique. Correlations between samples were studied by the Spearman rank correlation test. Both are non-parametric tests.

\section{Results}

EXPRESSION OF CD25 AND CD122 ON PERIPHERAL BLOOD LYMPHOCYTES

Tables 1 and 2 show the medians, first and third quartiles of CD25, CD122, CD124, and CD126 expression on PBL in patients with SLE and normal controls. Representative staining patterns of these cytokine receptors are shown in fig 1 . The percentage of PBL that were $\mathrm{CD} 25+$ was significantly higher in patients with SLE than in controls $(p<0.001)$. The relative density of CD25 molecules on CD25+ $\mathrm{PBL}$, as indicated by the mean channel number of fluorescent cells, was also significantly higher in patients with SLE $(p<0.05)$. Expression of CD25 was similar in controls and patients with active disease (SLEDAI >9). However, expression of CD25 in those with inactive disease (SLEDAI < 5) was significantly higher than in normal controls ( $\mathrm{p}<0.01$ ).

Conversely, a significantly greater proportion of PBL in normal controls were CD122+ than in patients with SLE $(p<0.05)$. Those patients with inactive disease (SLEDAI $<5)$ had a significantly lower percentage of CD122+ PBL than normal controls ( $p<$ $0.05)$. The difference between patients with active disease (SLEDAI > 9) and normal controls was not statistically significant (table 1). There was no difference in the antigenic level of CD122+ PBL between patients with SLE and controls. The difference in the number of CD122+ cells was greater in the CD122 $2^{\text {weak }}$ population (SLE $12.4 \% \quad v$ controls 19.4\%) than the CD122 $2^{\text {strong }}$ (SLE $1.8 \% v$ controls $2.4 \%$ ) population, although these differences were not statistically significant. The CD122 $2^{\text {strong }}$ cells were known to be natural killer cells. 

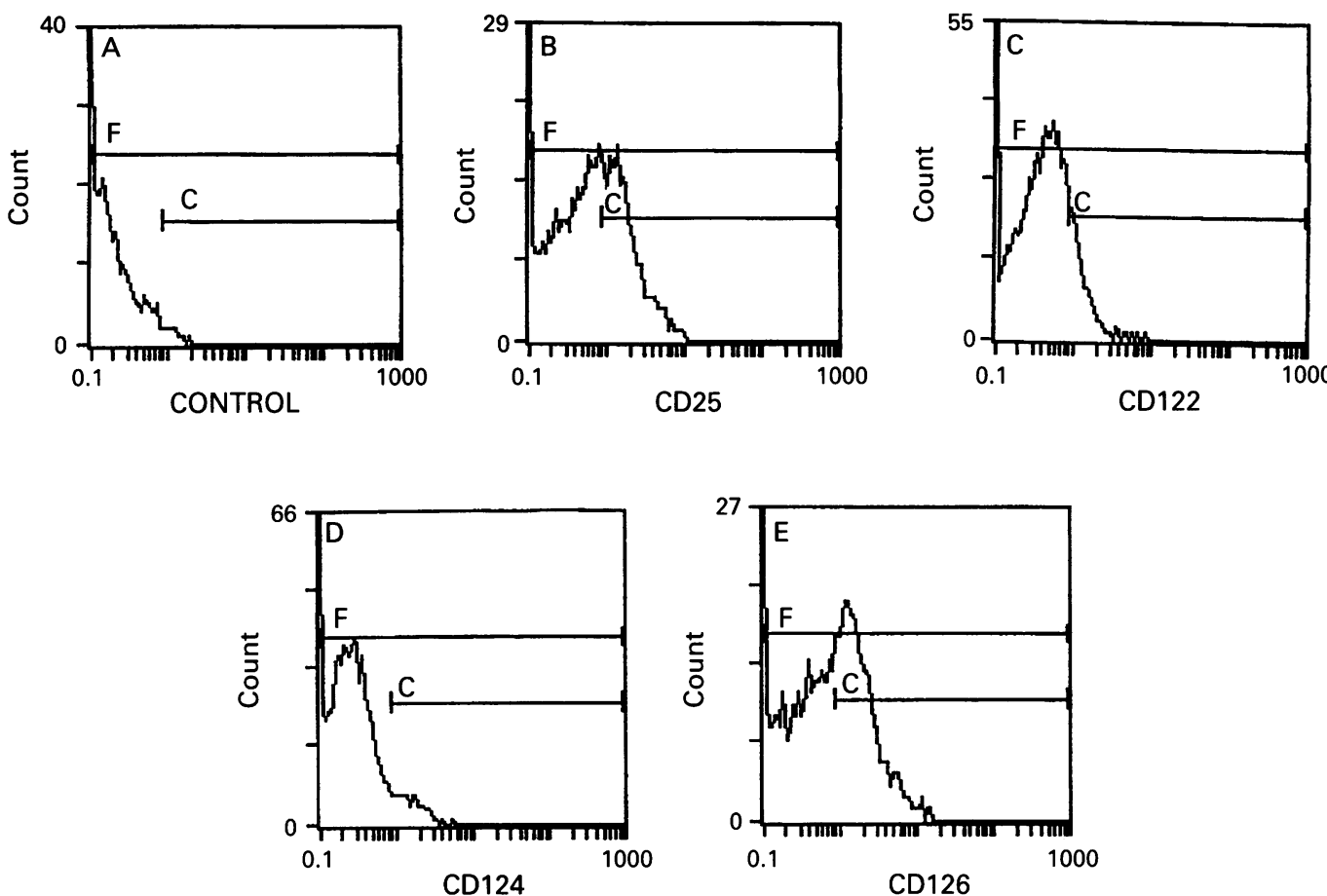

Figure 1 Expression of IL-2R,IL-4R and IL-6R on peripheral blood lymphocytes from patients with SLE (fluorescence level on $x$ axis in log scale; number of cells on y axis in linear scale). (A) Isotypic control; (B) IL-2R $\alpha$ chain (CD25); (C) IL-2R $\beta$ chain (CD122); (D) IL-4R (CD124); (E) IL-6R (CD126).

EXPRESSION OF CD124 AND CD126 ON PERIPHERAL BLOOD LYMPHOCYTES No significant difference was found -with regard to the expression of CD124 (IL-4R) and CD126 (IL-6R) on PBL in patients with SLE and normal controls.

CORRELATION OF CYTOKINE RECEPTOR

EXPRESSION WITH DISEASE ACTIVITY

Neither the percentage nor the relative antigenic level of $\mathrm{CD} 25, \mathrm{CD} 122, \mathrm{CD} 124$, or CD126 correlated with disease activity as scored by SLEDAI, C3 and C4 concentrations, anti-dsDNA level and absolute lymphocyte count.

\section{Discussion}

Interleukin-2 is an important cytokine produced by $T$ cells upon activation. It exerts numerous immunological effects by stimulating proliferation and lymphokine production by $T$ cells, $B$ cells and natural killer cells. Following activation, $\mathrm{T}$ cells also express the IL-2R and become responsive to IL-2. We show here that the percentage and the relative density of CD25 expressed on PBL is significantly higher in patients with SLE than in normal controls. This is consistent with several previous studies ${ }^{6-8}$ which reported an increase in CD25 expression on PBL of patients with SLE. In two of these reports, ${ }^{6} 7 \mathrm{CD} 25$ expression was significantly higher in patients with active disease than in those with inactive disease or in normal controls. The third study ${ }^{8}$ reported an increase in the expression of CD25 in patients with and without active disease, which suggests that there is no correlation between CD25 expression and disease activity. Our study sought to obtain paired data of CD25 expression and markers of disease activity and found no correlation between the two.
Furthermore, expression of CD25 on PBL in patients with SLE with active disease (SLEDAI > 9) was similar to that in normal subjects. These findings suggest increased CD25 expression on PBL may be a constitutive abnormality found only in patients with inactive SLE. Therefore, expression of CD25 cannot be used as a marker of disease progression in SLE.

Increased plasma concentrations of IL- 2 贾 have been reported in patients with SLE. ${ }^{13}$ This, together with increased expression of $\overline{ }$ CD25 on PBL, suggests that lymphocytes are in an activated state in vivo. However, stimulation of lymphocytes from patients with SLE with mitogens resulted in little increase in

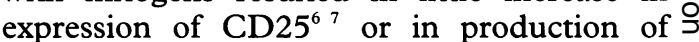
IL-2 ${ }^{2}$ when compared with stimulation of nor- $\frac{D}{O}$ mal lymphocytes. This in vitro $\mathrm{T}$ cell defect could reflect exhaustion of activated $\mathrm{T}$ cells. $N$ Ishida et al $^{14}$ demonstrated that the expression $N$ of high affinity IL-2R on these in vitro N activated lymphocytes was lower in cells obtained from patients with SLE. Furthermore, we found that there was no significant difference in expression of CD25 on PBL in $\stackrel{?}{?}$ patients with active disease and normal sub- $\frac{T}{T}$ jects. These results suggest that $T$ cells are $\stackrel{P}{\mathbb{P}}$ exhausted during active disease. Recent studies $\stackrel{\odot}{\oplus}$ have shown that in patients with SLE expres- \& sion of Fas is upregulated on blood lymphocytes ${ }^{15}$ and cultured lymphocytes undergo 8 apoptosis to a greater degree. ${ }^{16}$ Moreover, activation associated lymphocyte death has been demonstrated in HIV positive subjects. ${ }^{17}$ These $\stackrel{?}{?}$ observations suggest that cells activated in vivo, with upregulation of CD25 and Fas expression, in patients with active SLE may subsequently die via apoptosis.

IL-2R is a complex involving three distinct protein chains. ${ }^{18}$ The $\alpha$ chain (CD25) seems to 
be involved in the initial binding of IL-2, and is highly sensitive to upregulation by activation, while the $\beta$ and $\gamma$ chains are essential for signal transduction. The $\gamma$ chain is constitutively expressed in normal lymphocytes. ${ }^{19}$ In normal cell populations, the $\alpha$ and $\beta$ chains are expressed essentially in a reciprocal manner, although a small number of cells express detectable levels of both chains. ${ }^{20}$ This reciprocal relation between the $\alpha$ and $\beta$ chains was also demonstrated in the present study in lymphocytes from patients with SLE. Using radiolabelled IL-2 instead of monoclonal antibody, the expression of $\mathrm{p} 75$ on $\mathrm{T}$ cells of patients with SLE was found to be lower than in normal $\mathrm{T}$ cells. ${ }^{21}$ On phytohaemagglutinin stimulated blasts, expression of high affinity IL-2R was also lower in patients with SLE than normal controls, although there was no difference in the expression of CD25. ${ }^{14}$ This decrease in p75 expression suggests that the increased CD25 expression may not indicate a greater responsiveness to IL-2, but rather is a symptom of persisting activation.

The role of IL-4 and IL- 6 in the pathogenesis of SLE is not known. It has been reported that patients with SLE have higher circulating concentrations of IL -4 and IL $-6^{22}$ than normal controls. Furthermore, CD4+CD29+ T cells, which were the major $\mathrm{T}$ cells producing IL-6, were also increased in the circulation of patients with SLE. ${ }^{23}$ It is postulated that overexpression of these cytokines may contribute to the B cell hyperactivity and enhanced antibody synthesis characteristic of this autoimmune disease. In the present study, the level of expression of IL-4R and IL-6R on PBL of patients with SLE was no different from that in normal subjects. It would be interesting to know whether there is any difference in expression of these receptors on T, B and other lymphocyte subsets. Nagafuchi et $a l^{4}$ reported overexpression of IL-6R on B lymphocytes of patients with SLE. Further study on these cytokines and cytokine receptors should help to delineate the immunological abnormalities in SLE.

This study is supported by a research grant from the Council of Research and Conference Grant, Hong Kong.

1 Cuadrado MJ, Marubayashi M, Ortega C, Fernandez-Arcas N, Garcia-Cozar F, Pena J, et al. Relationship of IL-2, IL-2R (CD25+), soluble IL-2R and IL-4 with disease activity in SLE patients. Lupus 1993;2:257-60.

2 Alcocer-Varela J, Alarcon-Segovia D. Decreased production of interleukin-2 by cultured lymphocytes from patients with systemic lupus erythematosus. $\mathcal{f}$ Clin Invest 1982; 69:1388-92.

3 Huang YP, Miescher PA, Zubler RH. The interleukin-2 secretion defect in vitro in systemic lupus erythematosus is
reversible in rested cultured $T$ cells. $\mathcal{F}$ Immunol 1986; 137:3515-20.
4 Razziudin S, Al Janadi MA, Wabell AA. Soluble IL-2 receptor levels in serum and its relationship to T cell abnormality and clinical manifestations of the disease in patients with SLE. F Rheumatol 1991;18:831-6.

5 Ter-Borg EJ, Horst G, Limburg PC, Kallenberg CG. Changes in plasma levels of interleukin-2 receptor in relation to disease exacerbations and levels of anti-dsDNA and complement in systemic lupus erythematosus. Clin Exp Immunol 1990;82:21-6.

6 Wigfall DR, Sakai RS, Wallace DJ, Jordan SC. Interleukin-2 receptor expression in peripheral blood lymphocytes from systemic lupus erythematosus patients: relationship to systemic lupus erythematosus patients: relationship to
clinical activity. Clin Immunol Immunopathol 1982;47:35462 .

7 Alcocer-Varela J, Alarcon-Riqualme M, Laffon A, SachezMadrid F, Alarcon-Segovia AD. Activation markers on peripheral blood $\mathrm{T}$ cells from patients with active or inactive systemic lupus erythematosus. Correlation with proliferative responses and production of IL-2. I Autoimmun 1991;4:935-45.

8 Spronk PE, v.d. Gun BT, Limburg PC, Kallenberg CG. B cell activation in clinically quiescent systemic lupus erythematosus (SLE) is related to immunoglobulin levels, but not to levels of anti-dsDNA, nor to concurrent $\mathrm{T}$ cell activation. Clin Exp Immunol 1993;93:39-44.

9 Via CS, Handwerger BS. B cell and T cell function in systemic lupus erythematosus. Curr Opin Rheumatol 1993; 5:570-4.

10 Bombardier C, Gladman DD, Urowitz MB, Caron D, Chang CH. Derivation of the SLEDAI. A disease activity index for lupus patients. Arthritis Rheum 1992;35:630-40

11 Tan EM, Cohen AS, Fries JF, Masi AT, McShane DJ, Rothfield, et al. The 1982 revised criteria for the classification of systemic lupus erythematosus. Arthritis Rheum 1982, 25:1271-7.

12 Zola H, Flego L, Sheldon A. Detection of cytokine receptors by high-sensitivity immunofluorescence/flow cytometry. Immunobiology 1992;185:350-65.

13 Huang YP, Perrin LH, Miescher PA, Zubler RA. Correlation of $\mathrm{T}$ and $\mathrm{B}$ cell activities in vitro and serum IL-2 levels in systemic lupus erythematosus. 7 Immunol 1988; els in syste

14 Ishida H, Kumagai S, Umehara H, Sano H, Tagaya Y, Yodo $\mathrm{J}$, et al. Impaired expression of high affinity interleukin 2 receptor on activated lymphocytes from patients with systemic lupus erythematosus. F Immunol 1987;139:1070-4.

15 Amasaki Y, Kobayashi S, Takeda T, Ogura N, Jodo S, Nakabayashi T, et al. Up-regulated expression of Fas antigen (CD95) by peripheral naive and memory $\mathrm{T}$ cell gen (CD 95) by peripheral naive and memory $\mathrm{T}$ cell
subsets in patients with systemic lupus erythematosus subsets in patients with systemic lupus erythematosus Immunol 1995;99:245-50.

16 Emlen W, Niebur J, Kadera R. Accelerated in vitro apoptosis of lymphocytes from patients with systemic lupus erythematosus. F Immunol 1994;152:3685-92.

17 Janossy G, Borthwick N, Lomnitzer R, Medina E, Squire E Phillips AN, et al. Lymphocyte activation in HIV-1 infection. I. Predominant proliferative defects among $\mathrm{CD} 45 \mathrm{RO}+$ cells of the CD4 and CD8 lineages. AIDS 1993;7:613-24.

18 Taniguchi T, Minami Y. The IL-2/IL-2 receptor system: curret overview. Cell 1993;73:5-8.

19 Ishii N, Takeshita T, Kimura Y, Tada K, Kondo M, Nakamura $M$, et al. Expression of the IL-2 receptor $\gamma$ chain on various populations in human peripheral blood. In Immunol 1994;6: 1273-7.

20 Sheldon A, Flego L, Zola H. Coexpression of IL-2 recepto $\mathrm{p} 55$ and $\mathrm{p} 75$ by circulating blood lymphocytes. $\mathcal{f}$ Leukocyte Biol 1993;54:161-7.

21 Tanaka T, Saiki O, Negoro S, Igarashi T, Kuritani T, Hara $\mathrm{H}$, et al. Decreased expression of interleukin-2 binding molecules (p70/75) in T cells from patients with systemic lupus erythematosus. Arthritis Rheum 1989;32:552-9.

22 Linker-Isreali M, Deans RJ, Wallace DJ, Prehn J, OzeriChen T, Klinenber JR. Elevated levels of endogenous IL- 6 in systemic lupus erythematosus. A putative role in pathoin systemic lupus erythematosus. A putat
genesis. F Immunol 1991;147:117-23.

23 Al-Janadi M, Raziuddin S. B cell hyperactivity is a function of $T$ cell derived cytokines in systemic lupus erythematosus. F Rheumatol 1993;20:1885-91.

24 Nagafuchi H, Suzuki N, Mizushima A, Sakane T. Constitutive expression of IL-6 receptors and their role in the excessive B cell function in patients with systemic lupus erythematosus. F Immunol 1993;151:6525-34. 